\title{
Manual Therapy Versus Closed Kinematic Exercises-The Influence on the Range of Movement in Patients with Knee Osteoarthritis: A Pilot Study
}

\author{
Krystyna Hejdysz ${ }^{1}$, Jagoda Goślińska ${ }^{1, *}$ (D), Agnieszka Wareńczak ${ }^{1}{ }^{\circledR}$, Joanna Dudzińska ${ }^{1}$, \\ Ewa Adamczyk ${ }^{1}$, Paweł Sip ${ }^{1}$, Jarosław Gośliński ${ }^{2}{ }^{1}$, Piotr Owczarek ${ }^{2}$, Adam Woźniak ${ }^{2}$ \\ and Przemysław Lisiński ${ }^{1}$ (D) \\ 1 Department of Rehabilitation and Physiotherapy, University of Medical Sciences, 28 Czerwca 1956 Str., No 135/147, \\ 60-545 Poznań, Poland; krystynahejdysz@ump.edu.pl (K.H.); agnieszka.warenczak@ump.edu.pl (A.W.); \\ jdudzinska@ump.edu.pl (J.D.); ewaadamczyk@ump.edu.pl (E.A.); pawel.sip@ump.edu.pl (P.S.); \\ plisinski@vp.pl (P.L.) \\ 2 Aisens Sp. z o. o., Lubeckiego 23A, 60-348 Poznań, Poland; j.goslinski@aisens.co (J.G.); \\ p.owczarek@aisens.co (P.O.); a.wozniak@aisens.co (A.W.) \\ * Correspondence: jgoslinska@ump.edu.pl; Tel.: +48-506-586-111
}

Received: 5 October 2020; Accepted: 28 November 2020; Published: 1 December 2020

\begin{abstract}
Reduced range of motion is one of the main symptoms of knee osteoarthritis. These deficits are believed to have a negative impact on activities of daily living. The aim of the study was to examine how manual therapy and closed-chain kinematic exercises affect the range of motion in patients with knee osteoarthritis. Sixty-six patients with knee osteoarthritis were recruited and divided into three groups: manual therapy group, exercise group, and control group. The following parameters were evaluated before and after 10 days of rehabilitation: the range of motion in the open and closed kinematic chain using Orthyo sensors, pain intensity using Visual Analogue Scale (VAS), and the subjective functional assessment in Western Ontario and McMaster Universities Osteoarthritis Index (WOMAC). The results indicate an increase in the range of motion after manual therapy in the open chain test and an increase in the possible range of motion in the closed chain test in the exercise group. Both study groups showed significant improvement of WOMAC-assessed function and a significant decrease of VAS-assessed pain following rehabilitation. Manual therapy and exercise affect the range of motion in patients with knee osteoarthritis. When examining the range of motion, it is worth taking into account various biomechanical conditions.
\end{abstract}

Keywords: knee osteoarthritis; range of motion; manual therapy; exercises; inertial sensors

\section{Introduction}

Osteoarthritis $(\mathrm{OA})$ is a common condition reported in females more frequently than in males [1]. It typically affects joints in hands, feet, spine, knees, and hips [2]. Primary symptoms reported by patients suffering from osteoarthritis of the knee (OAK) include pain, stiffness, decreased mobility, and muscle weakness [1,3]. Clynes et al. [4] have proven that radiologically confirmed OAK is associated with problems with walking, self-care, and activities of daily living (ADLs). It has been shown by researchers that ADLs are affected not only by the pain reported but also by the range of motion [5]. The range of motion (ROM) may decrease due to pain, loss of elasticity in connective tissue structures, or bone abnormalities [6].

Most authors suggest that the range of 100-110 degrees in the knee is necessary for activities of daily living $[7,8]$. It has been noted, however, that a number of available studies evaluated functions, such as gait, climbing and descending stairs, i.e., activities that typically cannot be avoided or replaced $[7,8]$. 
However, there are a number of other activities that require a larger range of knee motion, such as getting into a bathtub, gardening, DIY, or changing socks. Some of these activities are commonly performed while squatting when the knee flexion range significantly exceeds 110 degrees. This was noted by Hyodo et al. who assessed hip, knee, and ankle angles required for various activities of daily living, such as dressing, using the toilet, bathing, picking up objects, and crouching [5]. It has been found that the knee flexion angle when getting into a standard bathtub was approximately 125 degrees, while trunk rotation during crouching required as much as 149 degrees [5]. Those who cannot achieve the required range of motion, no longer perform the activity or establish their own movement patterns, and this may cause other structures to be overloaded [9].

Lambert [10] suggests that activities of daily living more commonly involve closed kinematic chains or mixed sequences in which open and closed kinematic chains movements are alternately performed during different phases of an activity. More and more rehabilitation protocols include closed kinematic chain exercises; however, it is interesting to note that therapeutic efficacy is rarely evaluated based on closed kinematic chain tests only.

Treatment aiming to improve the range of motion in a joint depends on the cause of impairment. Major structural abnormalities in the joint frequently require surgical intervention [11]. Conservative treatment achieves satisfactory effects when impairment is due to other causes. The conservative treatment strategies to improve the range of motion include stretching exercises, passive exercises, active assisted exercises, manual therapy, and any physical procedures and exercises to alleviate pain and improve neuromuscular coordination which also improve the range of motion.

When reviewing the available study results, we have found that no research has been performed to determine the effect of specific therapeutic procedures on the range of motion and how they affect ADLs.

Therefore, this study aimed to answer the following questions:

1. Can we influence the range of movement within ten days of rehabilitation through manual therapy or synergistic movement lower limb exercises in the closed kinematic chain?

2. Which of the proposed physiotherapeutic methods better affects the range of movement?

3. Is testing in different biomechanical conditions useful for assessing the range of motion?

4. What are the further guidelines for a long-term physiotherapeutic protocol to improve OAK patients' range of movement?

Our study evaluated the short-term effect after 10 days of daily therapy, which was sufficient to evaluate certain tendencies and design further stages of rehabilitation showing greater effectiveness.

\section{Materials and Methods}

\subsection{Participants}

A randomized study was conducted in the Day Care Orthopedic and Rehabilitation Department, Teaching Hospital of the Poznań University of Medical Sciences between December 2018 and April 2019. Each patient had a physical examination along with an analysis of X-rays and patient history performed by a doctor. Out of a group of 147 patients with clinically confirmed osteoarthritis of the knees (Kellegren-Lawrence stage 1 or 2), 66 were enrolled based on inclusion and exclusion criteria. The mean age was 64 years (SD =5). There were 49 women and 17 men enrolled.

Exclusion criteria:

- advanced inflammatory osteoarthritis of the knee (Kellegren-Lawrence stage 3 or 4),

- patellofemoral disorders,

- neurological diseases,

- age below 55 or above 75 years,

- neoplastic disease,

- other rheumatic conditions (rheumatoid arthritis, ankylosing spondylitis, psoriatic spondylitis),

- sciatic neuralgia, 
- status post major lower limb trauma,

- $\quad$ status post lower limb surgery (e.g. arthroscopy, replacement arthroplasty),

- congenital defects and deformation of the lower limbs.

In spite of radiological signs of osteoarthritis, 22 patients participating in the study did not report knee pain during activities of daily living. Therefore, they were assigned to a control group. The other 44 patients who reported knee pain during activity and movement-related limitations of activities of daily living were randomly assigned to one of two groups on Day 1. Patients in the first group performed exercises (E) while those in the second group had manual therapy of the knees performed by a physical therapist (MT). The duration of a group-specific therapy in groups E and MT was 10 days. No therapy was administered in the control group (C). All patients were examined on Days 1 and 10 of the therapy.

The groups were similar in terms of age and sex. A $p$-value less than 0.05 was considered a statistically significant result. The subjects were aware of the study objectives and methodology and signed written consent for their participation. The study was approved by the Ethics Committee of the Poznań University of Medical Sciences and was consistent with the Declaration of Helsinki. The basic anthropometric data are listed in Table 1.

Table 1. Basic anthropometric data of the study population (Kruskal-Wallis test).

\begin{tabular}{cccccc}
\hline Variable & Group & Mean \pm SD & Median & Min-Max & $p$ \\
\hline \multirow{2}{*}{ age (years) } & E & $62.4 \pm 5.4$ & 62.0 & $55.0-75.0$ & \\
& MT & $65.2 \pm 3.1$ & 65.0 & $58.0-70.0$ & 0.13 \\
& C & $64.0 \pm 5.7$ & 63.0 & $55.0-75.0$ & \\
weight $(\mathrm{kg})$ & E & $78.0 \pm 12.2$ & 75.0 & $58.0-100.0$ & \\
& MT & $78.2 \pm 12.5$ & 75.0 & $58.0-105.0$ & 0.15 \\
& C & $71.1 \pm 12.2$ & 68.0 & $56.0-90.0$ & \\
\hline \multirow{2}{*}{ height $(\mathrm{m})$} & E & $1.68 \pm 0.08$ & 1.67 & $1.53-1.82$ & \\
& MT & $1.65 \pm 0.08$ & 1.64 & $1.55-1.81$ & 0.88 \\
& C & $1.68 \pm 0.07$ & 1.68 & $1.56-1.80$ & \\
\hline \multirow{3}{*}{ body mass index $(\mathrm{BMI})$} & E & $27.6 \pm 3.7$ & 27.1 & $20.4-36.1$ & \\
& MT & $28.6 \pm 4.3$ & 28.3 & $20.9-38.3$ & 0.40 \\
\hline
\end{tabular}

\subsection{Experimental Procedures and Instruments}

The patients were examined at the beginning and at the end of the therapy. This involved conducting two tests, three times each, using Orthyo sensors (one in the open and the other in the closed kinematic chain), completing the Western Ontario and McMaster University of Osteoarthritis Index (WOMAC) questionnaire (Polish translation), and pain assessment according to the Visual Analogue Scale (VAS).

\subsubsection{Range of Motion (Orthyo System)}

The patients were assigned to their groups and registered in the Orthyo online panel. Orthyo contains three parts: motion sensors, a mobile app, and an online panel. The sensors record 3 types of sensory data: magnetic field, acceleration, and velocity. After preliminary analysis, data are sent to the Orthyo app through Bluetooth Low Energy. Subsequently, all positions between the sensors in relation to each other are calculated and then stored in the online database. The Orthyo system has been discussed in detail in an article by Lisiński et al. [12].

Before the diagnostic examination, two motion sensors were attached to the patient's lower limb using elastic Velcro bands. The first one was attached to the lateral thigh area $15 \mathrm{~cm}$ from the trochanter of the femur, and the other on the anterior side of the lower leg, $5 \mathrm{~cm}$ distally from the tibial tuberosity. The maximum range of knee motion was measured using the sensors during two tests: 
1. Range of active knee motion: test in the open kinematic chain. The patient performed the test in the prone position with the lower limbs extended. The patient was requested to perform maximum knee flexion that would not elicit pain. The test was recorded by the motion sensors that calculated the difference between the initial and maximum angle (Figure 1).
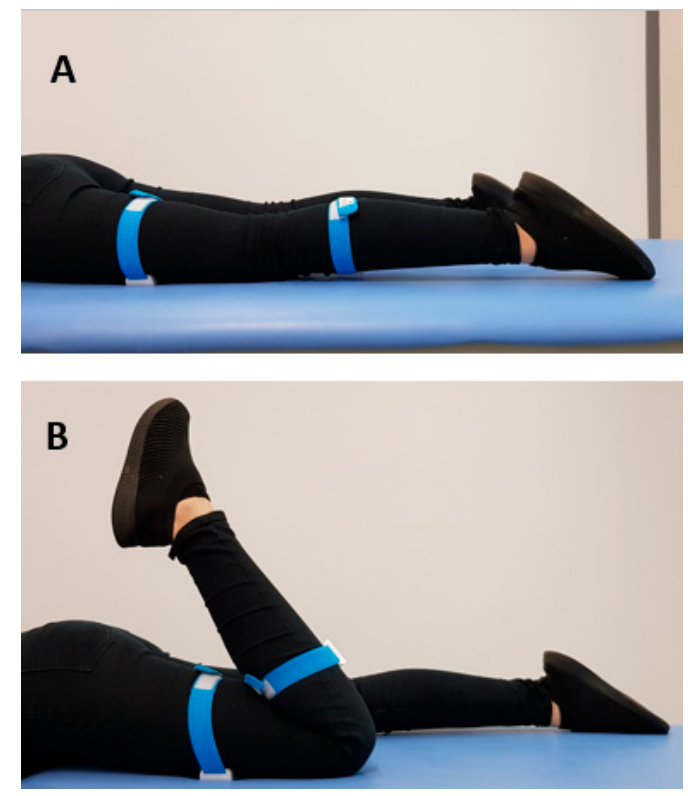

Figure 1. Measuring the range of motion in the open kinematic chain. (A) Starting position. (B) End position.

2. Maximum range of knee motion test while squatting. The patient performed the test in a standing position with the lower limbs hip-width apart. Subsequently, the patient was asked to squat as much as possible without feeling pain (Figure 2).
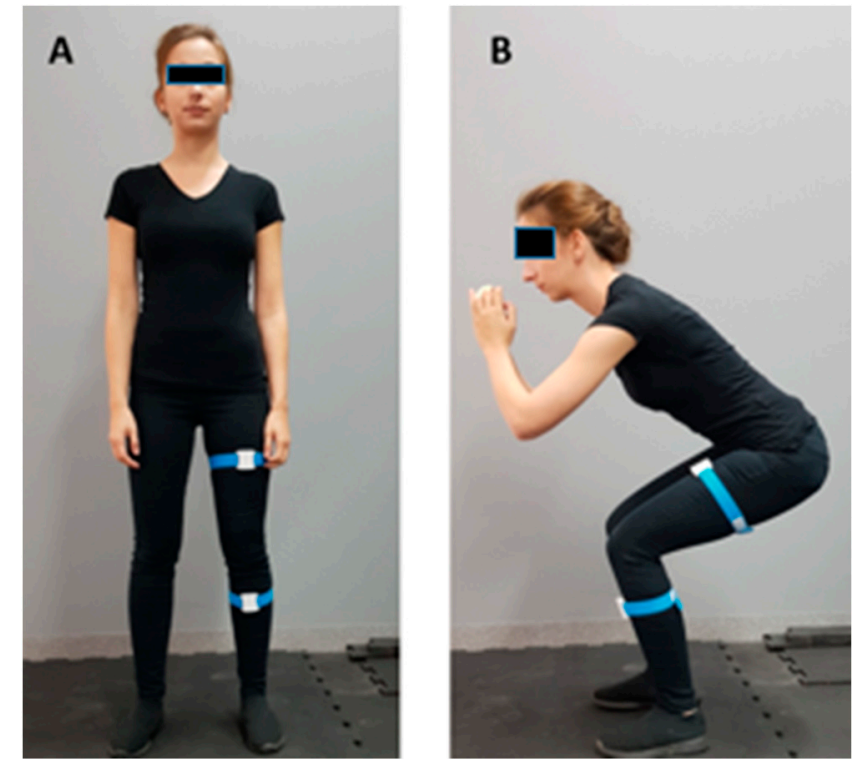

Figure 2. Measuring the range of motion in the closed kinematic chain. (A) Starting position. (B) End position. 
Both tests were performed three times for both lower limbs. Both limbs were similarly affected by the osteoarthritis in the patients, as confirmed by radiography. Therefore, differences between the right and left side were not included in the study.

\subsubsection{WOMAC}

WOMAC is used in the subjective functional evaluation of the knee [13]. It consists of 24 items divided into three categories: pain, stiffness, and physical function. Patients answered each question using a five-point scale. The maximum score was 96 points. A high WOMAC score indicates greater pain, stiffness, and functional limitations. Based on previous results [13], WOMAC is commonly used in research studies.

\subsubsection{VAS}

VAS is a subjective analogue scale used to evaluate the experienced level of pain [14]. The patient is asked to mark pain intensity between 0 and 100 points on a $100 \mathrm{~mm}$ horizontal line. Value of 0 means no pain, while value of 100 denotes the worst pain imaginable. The higher the score, the greater patient-reported pain. VAS is widely used in clinical studies.

\subsection{Intervention}

The MT group underwent patellar mobilization and massage of the soft tissues of the quadriceps femoris, adductor muscles, iliotibial tract, and biceps femoris. The therapeutic techniques included trigger point therapy, and functional and transverse friction massage. A set of synergistic motion exercises was developed for the patients assigned to group E.

These included: rolling the ball on the wall in the supine position (the patients were asked to have the knees and hips flexed at 90 degrees with the feet pressing on the ball-Figure 3) and balance disc exercises in the standing position (Figure 4). Both groups followed this therapeutic regimen for 10 consecutive days. No therapy was administered in the control group.
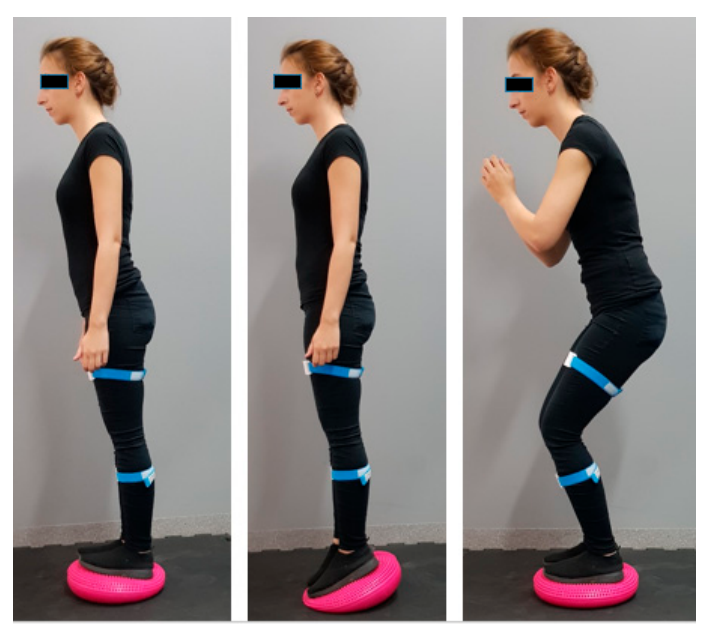

Figure 3. Exercises in the closed kinematic chain with ball.
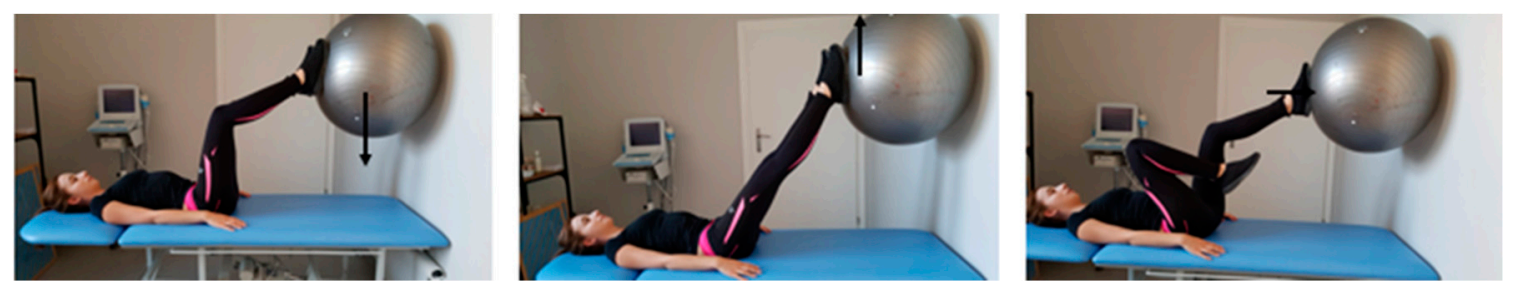

Figure 4. Closed kinematic chain exercises on the sensorimotor cushion. 


\subsection{Statistical Analysis}

Data analysis was performed using Statistica ${ }^{\mathrm{TM}}$ (TIBCO Software Inc., Palo Alto, CA, USA). version 13.1 Data listed include mean, median, standard deviation, and range. The Shapiro-Wilk test was used to evaluate normality of distribution. Student's t-tests and Wilcoxon rank tests were used to analyze and compare differences between results before and after therapy. Kruskal-Wallis and Mann-Whitney $\mathrm{U}$ tests were used to analyze differences between the three groups. Post hoc analysis was applied when statistically significant differences between the results were obtained. $p$-values of less than 0.05 were considered statistically significant and highlighted in tables using the blue color.

\section{Results}

\subsection{Range of Motion Measurements}

Test results of the range of active knee motion and squat depth were analysed. Intergroup comparison was calculated before and after 10-day therapy. In the test of the active range of motion, the groups differed both before and after therapy $(p=0.02)$. The post hoc analysis showed differences between the treatment groups and the control group. The flexion angle was greater in the control group by 11.3 and 8.7 degrees before and after therapy, respectively, in comparison with group E, and by 13 and 8.1 degrees before and after therapy, respectively, in comparison with group MT. The treatment groups were similar $(p=0.27)$. No intergroup differences were found in the squat depth test $(p=0.06)$. The flexion angle in the MT group significantly improved in the test of active range of motion $(p=0.01)$. The mean value and median increased by 5.7 and 5.6 degrees, respectively. In addition, the flexion angle significantly increased in the squat depth test in group $\mathrm{E}$. The mean value and median increased by 3.1 and 3.3 degrees, respectively. No statistically significant change in the squat test was found in the MT group. The values in group C did not significantly change in either test. The detailed results are listed in the table below (Table 2).

Table 2. Test results of the range of active knee motion and squat.

\begin{tabular}{|c|c|c|c|c|c|c|c|c|c|c|}
\hline \multirow{2}{*}{ Variable } & \multirow{2}{*}{ Group } & \multicolumn{4}{|c|}{ Before } & \multicolumn{4}{|c|}{ After } & \multirow{2}{*}{$\mathrm{p}^{3}$} \\
\hline & & Mean $\pm S D$ & Median & Range & $\mathrm{p}^{1}$ & Mean $\pm S D$ & Median & Range & $\mathrm{p}^{2}$ & \\
\hline \multirow{3}{*}{$\begin{array}{c}\text { Active } \\
\text { range } \\
\left({ }^{\circ}\right)\end{array}$} & $\mathrm{E}$ & $100.1 \pm 22.5$ & 106.4 & $19.7-141.2$ & \multirow{3}{*}{$\begin{array}{c}0.02 \\
0.27 *\end{array}$} & $103.5 \pm 22.2$ & 104.5 & $26.8-139.0$ & \multirow{3}{*}{$\begin{array}{c}0.04 \\
0.92 \text { * }\end{array}$} & 0.57 \\
\hline & MT & $98.4 \pm 21.2$ & 97.6 & 51.0-139.0 & & $104.1 \pm 16.8$ & 103.2 & $64.5-142.5$ & & $0.01 *$ \\
\hline & C & $111.4 \pm 15.5$ & 112.9 & $72.2-135.2$ & & $112.2 \pm 13.9$ & 115.9 & $85.9-141.2$ & & $0.73^{*}$ \\
\hline \multirow{3}{*}{$\begin{array}{l}\text { Squat } \\
\left(^{\circ}\right)\end{array}$} & $\mathrm{E}$ & $71.5 \pm 15.3$ & 73.2 & $33.2-95.8$ & \multirow{3}{*}{0.06} & $74.6 \pm 15.5$ & 76.5 & $38.1-100.6$ & \multirow{3}{*}{0.07} & $<0.01^{*}$ \\
\hline & MT & $81.1 \pm 19.2$ & 80.7 & $43.1-118.5$ & & $84.0 \pm 17.8$ & 81.5 & $45.9-115.0$ & & 0.12 * \\
\hline & $\mathrm{C}$ & $80.8 \pm 16.4$ & 82.6 & $52.7-122.8$ & & $82.1 \pm 16.1$ & 79.4 & $56.3-114.6$ & & 0.36 * \\
\hline
\end{tabular}

$\mathrm{p}^{1}$-intergroup (Kruskal-Wallis test or Mann-Whitney $U$ test); $\mathrm{p}^{2}$-intergroup post-treatment comparison (Kruskal-Wallis test or Mann-Whitney U test); $\mathrm{p}^{3}$-intragroup post-treatment comparison (dependent Student's $t$-test or * Wilcoxon signed-rank test).

\subsection{WOMAC}

The study compared the WOMAC results in all three groups before and after therapy (Table 3). The MT and E groups showed no statistically significant differences before the start of the study, and group $C$ had the lowest scores $(p<0.01)$. Marked improvement has been achieved in both treatment groups after the end of therapy (E: $p<0.01$, MT: $p=0.01$ ). Greater functional improvement has been achieved in the manual therapy group (decrease by 7.2 points) than in the exercise group (decrease by 5.4 points).

\subsection{VAS}

The study compared the VAS results in groups MT and E before and after therapy (Table 3). The group results were similar before the start of tests. Results in both groups significantly improved after the end of therapy $(p<0.01)$. The pain level significantly decreased in both groups without any significant difference between groups $\mathrm{E}$ and MT. 
Table 3. Results of Western Ontario and McMaster Universities Osteoarthritis Index (WOMAC) and Visual Analogue Scale (VAS) measures in Exercise (E), Manual Therapy (MT), and Control (C) groups of participants.

\begin{tabular}{|c|c|c|c|c|c|c|c|c|c|c|}
\hline \multirow{2}{*}{ Variable } & \multirow{2}{*}{ Group } & \multicolumn{4}{|c|}{ Before } & \multicolumn{4}{|c|}{ After } & \multirow{2}{*}{$\mathrm{p}^{3}$} \\
\hline & & Mean \pm SD & Median & Range & $\mathrm{p}^{1}$ & Mean \pm SD & Median & Range & $\mathrm{p}^{2}$ & \\
\hline \multirow{3}{*}{ WOMAC } & $\mathrm{E}$ & $41.8 \pm 138$ & 41.0 & $19.0-65.0$ & \multirow{3}{*}{$<0.01$} & $36.4 \pm 14.1$ & 39.0 & $08.0-58.0$ & \multirow{3}{*}{$<0.01$} & $<0.01$ \\
\hline & MT & $48.2 \pm 19.6$ & 47.0 & $2.0-81.0$ & & $41.0 \pm 28.8$ & 32.0 & $1.0-84.0$ & & 0.01 \\
\hline & $\mathrm{C}$ & $19.7 \pm 19.3$ & 12.5 & $2.0-78.0$ & & $19.0 \pm 20.6$ & 11.0 & $1.0-75.0$ & & $0.18^{*}$ \\
\hline \multirow{2}{*}{ VAS left } & $\mathrm{E}$ & $4.9 \pm 2.7$ & 5.5 & $0.0-10.0$ & \multirow{2}{*}{0.96} & $2.5 \pm 2.1$ & 2.5 & $0.0-6.0$ & \multirow{2}{*}{0.67} & $<0.01$ * \\
\hline & MT & $4.9 \pm 2.5$ & 5.0 & $0.0-8.0$ & & $3.1 \pm 2.7$ & 3.0 & $0.0-8.0$ & & $<0.01$ \\
\hline \multirow{2}{*}{ VAS right } & $\mathrm{E}$ & $5.0 \pm 2.6$ & 5.0 & $0.0-10.0$ & \multirow{2}{*}{0.09} & $2.8 \pm 2.0$ & 3.0 & $0.0-6.0$ & \multirow{2}{*}{0.34} & $<0.01$ \\
\hline & MT & $4.8 \pm 2.5$ & 5.0 & $0.0-8.0$ & & $3.2 \pm 2.7$ & 3.0 & $0.0-8.0$ & & $<0.01$ \\
\hline
\end{tabular}

$\mathrm{p}^{1}$-intergroup WOMAC and VAS pre-treatment comparison (Kruskal-Wallis test or Mann-Whitney U test); $\mathrm{p}^{2}$-intergroup WOMAC and VAS post-treatment comparison (Kruskal-Wallis test or Mann-Whitney U test); $\mathrm{p}^{3}$-intragroup WOMAC and VAS pre- and post-treatment comparison (dependent Student's $t$-test or * Wilcoxon signed-rank test)

\section{Discussion}

Highly diverse physiotherapeutic procedures are employed in patients with OAK. Our study evaluated how physiotherapeutic procedures conducted over 10 days would affect the range of knee motion. Two strategies were compared: synergistic movement exercises in the closed kinematic chain, and manual therapy. The results show that the range of motion improved in both procedures in two different tests performed in different evaluation conditions.

The range of motion in a joint is typically evaluated by various types of goniometers: electronic [15-17] or mechanical [18-20]. The disadvantage of such measurements is that they are usually conducted in a specific position of a patient to achieve as accurate the attachment of two goniometer arms as possible. Furthermore, mechanical goniometers introduce a significant measurement error and the results frequently depend on the examiner's subjective interpretation [21]. Our tests were based on inertial sensors to evaluate the range of motion irrespective of the position or type of the patient's activity. A number of other studies are available in which inertial sensors were used to evaluate the range of motion [12,22,23]. Approaches using camera-based motion capture systems are another method with similar measurement potential to inertial sensors. However, the measurements can be recorded only in specialized laboratories and with the required number of cameras, which is disadvantageous [21,24].

In our study we have demonstrated that the range of flexion evaluated in the conventional way in the prone position improved in patients after manual therapy. Other researchers have also reported an improved range of motion following manual therapy in patients with OAK [25-27], but there are also results available that show no such improvement [28]. It has been noted, however, that manual therapy includes both joint mobilization techniques and all techniques that affect soft tissues, so it is rather difficult to come to clear conclusions. Alkhawajah et al. [25] have reported a positive effect of short-term Maitland joint mobilization on the range of motion in patients with OAK, and Field [26] has shown positive effects following thigh muscle massage. Holla et al. [6] have revealed a clear correlation between pain intensity and a decreased range of motion in the joint. Studies show that pain may originate both in soft tissues [29] and in the joint itself [30], and it would seem that manual therapy that combines joint and soft-tissue related techniques should achieve the best results [27]. Therefore, our studies on a group of patients undergoing manual therapy involved not only joint-related techniques but also deep massage of the soft tissues of the thigh and lower leg, and that had a positive effect not only on the reported pain intensity but also on the range of motion. Similar results have been reported by Arshad et al. [27]. A number of studies evaluating the efficacy of manual therapy in combination with other procedures in terms of the range of motion [31,32] are available. Lizis et al. [31] have shown a significantly improved range of motion in a group undergoing manual therapy combined with cryotherapy. Other studies have reported an improved range of motion resulting from manual therapy in combination with exercises [32]. 
We have observed that improvement of the range of knee flexion in the manual therapy group was not significant in the maximum squat test. We believe that this could be due to an absent therapeutic intervention in the hip. A positive effect of such a therapy on knee motion has been shown experimentally by Cliborne et al. [33]. However, the squat depth is affected not only by the range of motion without pain, but also by coordination and muscle strength, which was certainly important in the manual therapy-only group.

The second group performed lower limb exercises in the closed kinematic chain. The main aim was to enhance the strength of the muscles acting on the whole lower limb, including the quadriceps, and thus improve the ROM. Huang et al. [19] basing on their results conclude that the decreased ROM is primarily caused by joint pain and a weak quadriceps. They believe that the tissues that surround the joint may become fibrotic, contracted, or shortened due to progressively lower mobility. Therefore, abnormalities develop in the articular capsule and the ROM decreases. We have seen in our results that the range of motion evaluated in the prone position in the open chain framework did not improve in such patients, but the maximum squat range did improve. We believe that our exercise regimen improved muscle strength and proprioception, and the patients were able to achieve a greater squat depth. It seems that the ability to maximize squat depth without pain will be much more useful to patients than the range of motion evaluated in the open chain in terms of activities of daily living. Other authors show that squatting is used when sitting down and getting up from a chair, crouching or using the toilet $[34,35]$. No positive effect in terms of the range of motion in the prone position has been observed in the exercise group, possibly because no techniques were applied to relax soft tissues and involve joint mobilization. Other exercises confirmed to be effective in improving the range of motion include stretching and strengthening exercises. It has been noted, however, that Mondam et al. [36] conclude that better effects on the range of motion are achieved when therapy includes proprioception training (similar to one in our study) than conventional strengthening exercises only.

WOMAC and VAS pain scores were used in most studies to evaluate improvement in the patient's clinical condition $[4,18,37,38]$. In our studies we have found significant functional improvement and decreased pain in the patients. The conclusions of Rhon et al. [39] are similar, as they reported marked improvement in patients following both manual therapy and exercises. According to Courtney et al. [30] impaired suppression of central nociceptive pathways occurs in the abnormal joint, which considerably improves with manual therapy. Positive effects of manual therapy, exercises or their combination on the improvement of these parameters have also been reported by other studies $[18,37,40,41]$.

The conclusions of other authors are confirmed based on our results in terms of the range of motion in both groups: a combination of therapy to relax excessively tight tissues and exercises most significantly improves the ROM, ADL scores and pain.

\section{Conclusions}

1. Manual 10-day therapy is beneficial for the range of knee motion in patients with OAK.

2. Synergistic 10-day lower limb movement exercises in the closed chain achieve greater squat depth without pain in patients with OAK.

3. Manual therapy in combination with exercises may potentially achieve a greater functional effect.

4. Measurements in different biomechanical conditions are useful when evaluating the range of motion.

5. Inertial sensors are a reliable and objective tool for evaluating the range of motion in different biomechanical conditions.

6. There is a need for further research aimed at assessing the long-term effect of the therapy.

\subsection{Clinical Implications}

Our pilot study shows that more effectiveness is achieved in OAK patients by combining manual therapy and exercise than by using only one type of treatment to improve the range of movement. 
Moreover, the results show that strength and proprioception training are also important in restoring the movement range in functional terms. We suspect that if the therapy took longer and combined both types of therapy the results would be better.

\subsection{Limitations}

To create a comprehensive algorithm of treatment, the whole spectrum of treatment methods of degenerative diseases should be considered not only in terms of physiotherapy. First of all, pharmacological treatment should be considered. Moreover, it would also be worth including other factors that could influence the results, such as daily physical activity beyond the applied physiotherapy or functional assessment of muscles, e.g., in the electromyographic examination.

Another limitation of our study is the short observation time. Degenerative disease is a chronic disease, therefore the evaluation of the longer-lasting therapy and, above all, the evaluation of the long-term effects of this treatment would be necessary.

Author Contributions: Conceptualization, J.G. (Jagoda Goślińska), A.W. (Agnieszka Wareńczak) and P.L.; Investigation, K.H., J.G. (Jagoda Goślińska), A.W. (Agnieszka Wareńczak), E.A. and P.S.; Methodology, K.H., J.G. (Jagoda Goślińska), A.W. (Agnieszka Wareńczak), E.A. and P.S.; Software, J.G. (Jarosław Gośliński), P.O. and A.W. (Adam Woźniak); Supervision, P.L.; Writing—original draft, K.H. and J.G. (Jagoda Goślińska); Writing—review \& editing, J.D. All authors have read and agreed to the published version of the manuscript.

Funding: The APC was funded from the financial resources of Poznan University of Medical Sciences designated for the statutory activities of the Rehabilitation Clinic.

Conflicts of Interest: The authors declare no conflict of interest.

\section{References}

1. Park, E.; Park, H.-R.; Choi, E.-S. Barriers to and Facilitators of Physical Activity among Korean Female Adults with Knee Osteoarthritis and Comorbidity: A Qualitative Study. Healthcare 2020, 8, 226. [CrossRef]

2. Xu, H.; Zhao, G.; Xia, F.; Liu, X.; Gong, L.; Wen, X. The diagnosis and treatment of knee osteoarthritis: A literature review. Int. J. Clin. Exp. Med. 2019, 12, 4589-4599.

3. Uchio, Y.; Enomoto, H.; Ishida, M.; Tsuji, T.; Ochiai, T.; Konno, S. Safety and efficacy of duloxetine in Japanese patients with chronic knee pain due to osteoarthritis: An open-label, long-term, Phase III extension study. J. Pain Res. 2018, 11, 1391-1403. [CrossRef] [PubMed]

4. Clynes, M.A.; Jameson, K.A.; Edwards, M.H.; Cooper, C.; Dennison, E.M. Impact of osteoarthritis on activities of daily living: Does joint site matter? Aging Clin. Exp. Res. 2019, 31, 1049-1056. [CrossRef] [PubMed]

5. Hyodo, K.; Masuda, T.; Aizawa, J.; Jinno, T.; Morita, S. Hip, knee, and ankle kinematics during activities of daily living: A cross-sectional study. Braz. J. Phys. Ther. 2017, 21, 159-166. [CrossRef] [PubMed]

6. Holla, J.F.M.; Steultjens, M.P.M.; van der Leeden, M.; Roorda, L.D.; Bierma-Zeinstra, S.M.A.; den Broeder, A.A.; Dekker, J. Determinants of range of joint motion in patients with early symptomatic osteoarthritis of the hip and/or knee: An exploratory study in the CHECK cohort. Osteoarthr. Cartil. 2011, 19, 411-419. [CrossRef]

7. Jevsevar, D.S.; Riley, P.O.; Hodge, W.A.; Krebs, D.E. Knee Kinematics and Kinetics During Locomotor Activities of Daily Living in Subjects with Knee Arthroplasty and in Healthy Control Subjects. Physical. Ther. 1993, 73, 229-239. [CrossRef]

8. Andriacchi, T.P.; Andersson, G.; Fermier, R.; Stern, D.; Galante, J. A study of lower-limb mechanics during stair-climbing. J. Bone Jt. Surg. Am. Vol. 1980, 62, 749-757. [CrossRef]

9. Stevermer, C. Functional Movement Assessment for Individuals with Knee Osteoarthritis. Ph.D. Thesis, Iowa State University, Ames, IA, USA, 2010.

10. Lambert, S.J. A Comparison of Closed v\& Open Kinetic Chain Exercises in the Rehabilitation of Anterior Cruciate Ligament Injuries. An Honors Thesis, Ball State University, Muncie, Indiana, 1998; pp. 1-15.

11. Skou, S.T.; Roos, E.M.; Laursen, M.B.; Rathleff, M.S.; Arendt-Nielsen, L.; Rasmussen, S.; Simonsen, O. Total knee replacement and non-surgical treatment of knee osteoarthritis: 2-year outcome from two parallel randomized controlled trials. Osteoarthr. Cartil. 2018, 26, 1170-1180. [CrossRef] [PubMed] 
12. Lisiński, P.; Wareńczak, A.; Hejdysz, K.; Sip, P.; Gośliński, J.; Owczarek, P.; Jonak, J.; Goślińska, J. Mobile Applications in Evaluations of Knee Joint Kinematics: A Pilot Study. Sensors 2019, 19, 3675. [CrossRef] [PubMed]

13. Jogi, P.; Overend, T.J.; Spaulding, S.J.; Zecevic, A.; Kramer, J.F. Effectiveness of balance exercises in the acute post-operative phase following total hip and knee arthroplasty: A randomized clinical trial. SAGE Open Med. 2015, 3. [CrossRef] [PubMed]

14. Brokelman, R.B.G.; Haverkamp, D.; van Loon, C.; Hol, A.; van Kampen, A.; Veth, R. The validation of the visual analogue scale for patient satisfaction after total hip arthroplasty. Eur. Orthop. Traumatol. 2012, 3, 101-105. [CrossRef] [PubMed]

15. Hu, X.; Lai, Z.; Wang, L. Effects of Taichi exercise on knee and ankle proprioception among individuals with knee osteoarthritis. Res. Sports Med. 2020, 28, 268-278. [CrossRef]

16. Lin, D.-H.; Lin, Y.-F.; Chai, H.-M.; Chai, H.-M.; Han, Y.-C.; Jan, M.-H. Comparison of proprioceptive functions between computerized proprioception facilitation exercise and closed kinetic chain exercise in patients with knee osteoarthritis. Clin. Rheumatol. 2007, 26, 520-528. [CrossRef]

17. Kumar, S.; Kumar, A.; Kumar, R. Proprioceptive training as an adjunct in osteoarthritis of knee. J. Musculoskelet. Res. 2013, 16, 1350002. [CrossRef]

18. Sekir, U.; Gür, H. A multi-station proprioceptive exercise program in patients with bilateral knee osteoarthrosis: Functional capacity, pain and sensoriomotor function. A randomized controlled trial. J Sports Sci. Med. 2005, 4, 590-603.

19. Huang, D.; Wang, H.-G.; Wu, W.-Z.; Zhang, H.-R.; Lin, H. Functional and aesthetic results of immediate reconstruction of traumatic thumb defects by toe-to-thumb transplantation. Int. Orthop. 2011, 35, 543-547. [CrossRef]

20. Abdelazeem, F.; Nambi, G.; Elnegamy, T. Comparative study on Virtual Reality Training (VRT) over Sensory Motor Training (SMT) in Unilateral Chronic Osteoarthritis-A Randomized Control Trial. Int. J. Med. Res. Health Sci. 2016, 5, 7-16.

21. Sobel, D.; Kwiatkowski, J.; Ryt, A.; Domzal, M.; Jedrasiak, K.; Janik, L.; Nawrat, A. Range of Motion Measurements Using Motion Capture Data and Augmented Reality Visualisation. In Proceedings of the Computer Vision and Graphics, Warsaw, Poland, 15-17 September 2014; Chmielewski, L.J., Kozera, R., Shin, B.-S., Wojciechowski, K., Eds.; Springer International Publishing: Cham, Switzerland, 2014; pp. 594-601.

22. Sharma, B.M.; Vidhya, S.; Kumar, N. System for measurement of joint range of motion using inertial sensors. Biomed. Res. 2017, 3699-3704.

23. Fennema, M.C.; Bloomfield, R.A.; Lanting, B.A.; Birmingham, T.B.; Teeter, M.G. Repeatability of measuring knee flexion angles with wearable inertial sensors. Knee 2019, 26, 97-105. [CrossRef]

24. Van der Kruk, E.; Reijne, M.M. Accuracy of human motion capture systems for sport applications; state-of-the-art review. Eur. J. Sport Sci. 2018, 18, 806-819. [CrossRef]

25. Alkhawajah, H.A.; Alshami, A.M. The effect of mobilization with movement on pain and function in patients with knee osteoarthritis: A randomized double-blind controlled trial. BMC Musculoskelet. Disord. 2019, 20, 452. [CrossRef]

26. Field, T.; Diego, M.; Gonzalez, G.; Funk, C.G. Knee arthritis pain is reduced and range of motion is increased following moderate pressure massage therapy. Complement. Ther. Clin. Pract. 2015, 21, 233-237. [CrossRef] [PubMed]

27. Arshad, N.; Gondal, M.I.; Qamar, M.M.; Basharat, A. Download citation of Manual therapy can be a potential therapy in knee osteoarthritis. Saudi J. Sports Med. 2018, 18, 18-21.

28. Perlman, A.I.; Ali, A.; Njike, V.Y.; Hom, D.; Davidi, A.; Gould-Fogerite, S.; Milak, C.; Katz, D.L. Massage Therapy for Osteoarthritis of the Knee: A Randomized Dose-Finding Trial. PLoS ONE 2012, 7, e30248. [CrossRef]

29. Dor, A.; Kalichman, L. A myofascial component of pain in knee osteoarthritis. J. Bodyw. Mov. Ther. 2017, 21, 642-647. [CrossRef] [PubMed]

30. Courtney, C.A.; Steffen, A.D.; Fernández-de-Las-Peñas, C.; Kim, J.; Chmell, S.J. Joint Mobilization Enhances Mechanisms of Conditioned Pain Modulation in Individuals with Osteoarthritis of the Knee. J. Orthop. Sports Phys. Ther. 2016, 46, 168-176. [CrossRef] [PubMed]

31. Lizis, P.; Manko, G.; Kobza, W.; Para, B. Manual Therapy with Cryotherapy versus Kinesiotherapy with Cryotherapy for Knee Osteoarthritis: A Randomized Controlled Trial. Altern. Ther. Health Med. 2019, 25, $40-45$. 
32. Hameed, R.; Waqas, M.; Akhtar, F.; Robinson, J.; Niazi, A. Mahmood Effect of Manual Therapy on Knee Osteoarthritis (OA) Pain, A Randomized Control Trial. Int. J. Res. Stud. Med. Health Sci. 2017, 2, 19-22. [CrossRef]

33. Cliborne, A.V.; Wainner, R.S.; Rhon, D.I.; Judd, C.D.; Fee, T.T.; Matekel, R.L.; Whitman, J.M. Clinical hip tests and a functional squat test in patients with knee osteoarthritis: Reliability, prevalence of positive test findings, and short-term response to hip mobilization. J. Orthop. Sports Phys. Ther. 2004, 34, 676-685. [CrossRef] [PubMed]

34. Vecchio, L.D. The health and performance benefits of the squat, deadlift, and bench press. MOJYPT 2018, 3, 40-47. [CrossRef]

35. Fox, J.T.; Hefzy, M.S. Knee and Ankle Biomechanics during Squatting with Heels on and Off of the Ground, with and without Weight Shifting. Bone Muscle 2019, 2, 1-9.

36. Mondam, S.; Babu, S.; Kumar, R.; Prakash, J. A Comparative Study of Proprioceptive Exercises versus Conventional Training Program on Osteoarthritis of Knee. RJRS 2012, 1, 2277-2502.

37. AdemolaGbiri, C. Comparative Efficacy of Open-chain and Close-chain Kinematics on Proprioception, Muscles' Strength and Functional Performances in Individual with Knee Osteoarthritis. Occup. Med. Health Aff. 2013, 1, 1-5. [CrossRef]

38. Reid, D.A.; McNair, P.J. Effects of an acute hamstring stretch in people with and without osteoarthritis of the knee. Physiotherapy 2010, 96, 14-21. [CrossRef]

39. Rhon, D.; Deyle, G.; Gill, N.; Rendeiro, D. Manual physical therapy and perturbation exercises in knee osteoarthritis. J. Man. Manip. Ther. 2013, 21, 220-228. [CrossRef]

40. Abbott, J.H.; Usiskin, I.M.; Wilson, R.; Hansen, P.; Losina, E. The quality-of-life burden of knee osteoarthritis in New Zealand adults: A model-based evaluation. PLoS ONE 2017, 12, e0185676. [CrossRef]

41. Deyle, G.D.; Allison, S.C.; Matekel, R.L.; Ryder, M.G.; Stang, J.M.; Gohdes, D.D.; Hutton, J.P.; Henderson, N.E.; Garber, M.B. Physical therapy treatment effectiveness for osteoarthritis of the knee: A randomized comparison of supervised clinical exercise and manual therapy procedures versus a home exercise program. Phys. Ther. 2005, 85, 1301-1317. [CrossRef]

Publisher's Note: MDPI stays neutral with regard to jurisdictional claims in published maps and institutional affiliations.

(C) 2020 by the authors. Licensee MDPI, Basel, Switzerland. This article is an open access article distributed under the terms and conditions of the Creative Commons Attribution (CC BY) license (http://creativecommons.org/licenses/by/4.0/). 